Publicacions Matemàtiques, Vol 35 (1991), 507-516.

\title{
INTERPOLATING SEQUENCES AND THE NEVANLINNA PICK PROBLEM
}

\author{
ARNE STRAY
}

\begin{abstract}
The extremal solutions to the Nevanlinna Pick problem are studied. If there is more than one solution, Nevantinna showed that all extremal solutions are inner functions. With some extra information on the interpolation data we find that the extremal solutions are Blaschke products whose zeroes form a finite union of interpolating sequences.
\end{abstract}

\section{Introduction}

Let $U$ denote the analytic functions bounded by one in the open unit disc $D$. Consider the Nevanlinna Pick interpolation problem

$$
(N P): f\left(z_{n}\right)=w_{n}, n=1,2, \ldots, f \in U
$$

In his work on this problem, R. Nevanlinna [6], demonstrated the importance of the so called vertevorrat corresponding to $(N P)$ :

$$
\Delta(z)=\{f(z): f \in E\}, z \in D \backslash\left\{z_{n}\right\}
$$

where $E$ is the set of solutions to $(N P)$.

If $E$ contains more than one function, Nevanlinna proved that $\Delta(z)$ is a disc. Moreover, he showed that each boundary value of $\Delta(z)$ is attained by a unique function from $E$, and that the class of solutions $I$ satisfying

$$
I(z) \in \partial \Delta(z)
$$

is independent of $z$. Any solution of $(N P)$ satisfying (1), will be called extremal. An important discovery by Nevanlinna is that every extremal solution is an inner function if $E$ is not a singleton. ([6, Satz 7] or [2, page 172]).

Let $\rho(z)$ denote the radius of $\Delta(z)$. It is not hard to see that each extremal solution being inner is equivalent to

$$
\lim _{r \rightarrow 1} \rho\left(r e^{i \theta}\right)=1
$$


for almost all $\theta \in[0,2 \pi)$ with respect to linear measure.

More information about the extremal solution to $(N P)$ may be obtained if one can improve on (2). Let $\Pi$ denote the Blaschke product corresponding to $\left\{z_{n}\right\}$ :

$$
\Pi(z)=\Pi_{n} \frac{\left|z_{n}\right|}{z_{n}} \frac{z_{n}-z}{1-\bar{z}_{n} z} .
$$

The main results of this work can be described as follows: For a large class of problems $(N P)$ it is shown that $\rho(z) \rightarrow 1$ if $|\Pi(z)| \rightarrow 1$.

Using this information about $\rho$, we assume that $\Pi$ factors into $\Pi=\Pi_{1}, \ldots, \Pi_{N}$ where the zeroes of $\Pi_{k}, 1 \leq k \leq N$ is an interpolating sequence (see definition below). Under various conditions on $\left\{w_{n}\right\}$ we prove that any extremal solution to $(N P)$ is a Blaschke product admitting a similar factorization.

This work is a continuation of $[9]$ and [10]. We use the book [2] by J. Garnett as a reference for the theory of the classical Hardyspaces $H^{p}, 0<p \leq \infty$ in $D$. In particular $H^{\infty}$ denotes the Banach space of all bounded analytic functions in $D$ with norm

$$
\|f\|_{\infty}=\sup \{|f(z)|: z \in D\} .
$$

We also have $\|f\|_{\infty}=\operatorname{ess} \sup _{0 \leq \theta<2 \pi}\left|f\left(e^{i \theta}\right)\right|$ where $f\left(e^{i \theta}\right)=\lim _{r \rightarrow 1} f\left(r e^{i \theta}\right)$ exists allmost everywhere $d \theta$ in light of Fatous theorem ([2, page 29]).

The sequence $\left\{z_{n}\right\} \subset D$ is called interpolating for $H^{\infty}$ if the problem $f\left(z_{n}\right)=$ $w_{n}, n=1,2, \ldots$ is solvable with $f \in H^{\infty}$, for any bounded sequence $\left\{w_{n}\right\}$. In [1], L. Carleson proved that $\left\{z_{n}\right\}$ is interpolating if and only if

$$
\inf _{n \neq m} \sigma\left(z_{n}, z_{m}\right) \geq \delta>0 \quad\left(\sigma(a, b)=\left|\frac{a-v}{1-\bar{v} a}\right|\right)
$$

and

$\left(C_{2}\right)$ : The measure $\sum\left(1-\left|z_{n}\right|\right) \delta_{z_{n}}$ is a Carleson measure, where $\delta_{z_{n}}$ denotes the point mass at $z_{n}$.

Condition $\left(C_{2}\right)$ implies that if $f$ belongs to the Hardy space $H^{1}$, then

$$
\sum\left|f\left(z_{n}\right)\right|\left(1-\left|z_{n}\right|\right)<\infty
$$

See $[\mathbf{2}$, page 63 and 287$]$ for details.

A Blaschke product whose zeros form an interpolating sequence, will be called an interpolating Blaschke product.

\section{Some theorems}

The problem (NP) will be called semiscaled if there is $f \in H^{\infty}$ such that $\|f\|_{\infty}<1$ and $f\left(z_{n}\right)=w_{n}, n \geq N$, for some $N$. If one can choose $N=1$, we say that $(N P)$ is a scaled problem. As in $1, \Pi$ denotes the Blaschke product corresponding to $\left\{z_{n}\right\}$. With this notation we have 
Theorem 1. Suppose the Nevanlinna Pick problem (NP) is semiscaled and has more than one solution. Then $\rho(z) \rightarrow 1$ if $|\Pi(z)| \rightarrow 1$, where $\rho(z)$ is the radius of the disc $\Delta(z)=\{f(z): f$ solves $(N P)\}$.

Remark. The hypothesis that $(N P)$ is semiscaled, can not be dropped. A. Nicolau recently found an example [7, p. 93], where $\left\{z_{n}\right\} \subset(0,1)$ is interpolating and $(N P)$ has more than one solution, such that $\left|\Pi\left(\xi_{k}\right)\right| \rightarrow 1$ while $\rho\left(\xi_{k}\right) \rightarrow 0$, for some sequence $\left\{\xi_{k}\right\} \subset(0,1)$.

Suppose $z_{n} \rightarrow 1$ within some cone included in $D$. Then we say that $\left\{z_{n}\right\}$ converges nontangentially to 1 .

Theorem 2. Suppose $z_{n} \rightarrow 1$ nontangentially and that the Nevanlinna Pick problem $(N P)$ with data $\left\{z_{n}\right\},\left\{w_{n}\right\}$ is semiscaled. Let I be any extremal solution to (NP). If $\Pi$ factors into finitely many interpolating Blaschke products, then $I$ is a Blaschke product admitting a similar factorization. Moreover, the zeroes of I converge to 1 nontangencially.

Our final result is only a slight extension of Theorem 1 in [9]. We include it here because of its relevance to recent work by $\mathrm{T}$. Nazaki in [5].

Theorem 3. Let $\left\{z_{n}\right\}$ be a finite union of interpolating sequences. There is a number $r>0$ depending only on $\left\{z_{n}\right\}$ with the followning property: Let $I$ be any extremal solution to a Nevanlinna Pick problem $(N P)$ with data $\left\{z_{n}\right\},\left\{w_{n}\right\}$. Suppose that there is $f \in H^{\infty}$ such that $\|f\|_{\infty} \leq r$ and $f\left(z_{n}\right)=w_{n}$ if $n$ is sufficiently large. Then I factors into finitely many interpolating Blaschke products.

\section{Proofs}

The method of proof has two main ingredients originating in the interpolation theory for $H^{\infty}$. We combine the classical work of R. Nevanilinna [5] by more recent ideas largely due to L. Carleson ([1], [2, ch. VII]).

If $(N P)$ has more than one solution, $\mathrm{R}$. Nevanlinna found analytic functions $P, Q, R$ and $S$ in $D$, such that all solutions to $(N P)$ are given by

$$
E=\left\{\frac{P-Q w}{R-S w}: w \in U\right\}
$$

The functions $P, Q, R$ and $S$ are normalized such that $P S-R Q=\Pi$. Fixing $z$ in $D \backslash\left\{z_{n}\right\}$ we see that $\Delta(z)=\{f(z): f \in E\}$ is the image of the closed unit disc under the Möbius transformation

$$
\Theta_{z}: w \rightarrow \frac{P(z)-Q(z) w}{R(z)-S(z) w}
$$


The extremal solutions to $(N P)$ are precisely the functions $I_{\alpha}$ corresponding to $w \equiv e^{i \alpha}, 0 \leq \alpha<2 \pi$. The radius $\rho(z)$ of $\Delta(z)$ is easily computed to be

$$
\rho(z)=\frac{|\Pi(z)|}{|R(z)|^{2}-|S(z)|^{2}} .
$$

We start by collecting some more or less well known results related to Nevanlinnas formula.

Lemma 1. Suppose the Nevanlinna Pick problem (NP) has more than one solution. Then $P, Q, R, S$ and $\rho$ have the following properties:

(i) $R^{-1} \in U$

(ii) $\max \{|P(z)|,|Q(z)|,|S(z)|\} \leq|R(z)|, z \in D$

(iii) $\rho(z) \leq|\Pi(z)|, z \in D$

(iv) If (NP) involves only fnitely many data, then $\rho(z) \geq 1-C(1-|z|), z \in$ $D$, for some constant $C>0$.

(v) If $(N P)$ is scaled, then $R \in H^{2}(D)$ and $(1-\lambda)|\Pi(z)| \leq \rho(z)$, $z \in D$, for some constant $\lambda \in(0,1)$.

In the concluding remarks following the proofs, we shall indicate how Lemma 1 follows from Nevanlinna's original paper $[6]$ and more recent results.

Proof of Theorem 1: We first use a deep result due to $P$. Jones to prove Theorem 1 if $(N P)$ is scaled. So we assume $(N P)$ is solved by some $f \in H^{\infty}$ such that $\|f\|_{\infty} \leq \lambda<1$. Given $\epsilon \in(0,1)$, let $r=1-\epsilon$ and choose $t \in(r, 1)$ such that $\sigma(r, t)>1-\epsilon$. Fix $a, b \in D$ such that $|\Pi(a)| \leq r,|\Pi(b)| \geq t$. By Schwarz lemma ([2, page 2]).

$$
\sigma(a, b) \geq \sigma(\Pi(a), \Pi(b)) \geq \sigma(r, t)>1-\epsilon .
$$

Hence if we define $\varphi \in H^{\infty}$ by $\varphi(z)=\frac{z-b}{1-\bar{b}_{z}}$, we have

$$
\max \{|\varphi(z)|,|\Pi(z)|\}>1-\epsilon
$$

for any $z \in D$. By Theorem 1 in P. Jones paper [4], there are functions $g_{1}, g_{2} \in H^{\infty}$ such that

$$
\Pi g_{1}+\varphi g_{2} \equiv 1
$$

and

$$
\left|g_{1}(z)\right|+\left|g_{2}(z)\right| \leq 1+A(\epsilon), z \in D
$$

where $A(\epsilon) \rightarrow 0$ if $\epsilon \rightarrow 0$. If $\epsilon$ is so small that $(1+A(\epsilon)) \lambda<1$, it follows that

$$
F_{w}=f \varphi g_{2}+w \Pi g_{1}
$$

solves $(N P)$ whenever $|w| \leq(1+A(\epsilon))^{-1}$. This means that $\rho(b)>(1-\epsilon)(1+$ $A(\epsilon))^{-1}$ if $|\Pi(b)|>I-t$, where $t=t(\epsilon)$ depends only on $\epsilon$. 
To prove Theorem $I$ in general, let us consider an expanded problem $(N P)_{1}$ obtained by adding finitely many constraints to $(N P)$. If a typical solutions to $(N P)$ is given by Nevanlinnas formula (4), we require in addition

$$
\frac{P\left(\xi_{k}\right)-Q\left(\xi_{k}\right) w\left(\xi_{k}\right)}{R\left(\xi_{k}\right)-S\left(\xi_{k}\right) w\left(\xi_{k}\right)}=\eta_{k}, 1 \leq k \leq N
$$

Then (5) defines a finite Nevanlinna Pick problem $(N P)_{2}$ for the unknown function $w \in U$. By assumption, it has more than one solution.

Corrresponding to $(N P)$ we have defined $\Delta(z), \rho(z)$ and the Möbius transformation $\Theta_{z}$. For $i=1,2$ we denote the same objects for $(N P)_{i}$ by $\Delta_{i}(z), \rho_{i}(z)$ and $\Theta_{z}^{i}$ respectively. If $c(z)$ denotes the center of $\Delta(z)$, it is well known ( $[9$, page 473]) that.

$$
c(z)=\Theta_{z}(\bar{a}(z))
$$

where $a(z)=\frac{S(z)}{R(z)}$. Let $S_{i}$ denote the image of the unit circle under the transformation $\Theta_{z}^{i}$. Then since $\Delta_{1}(z) \subset \Delta(z)$, we have

$$
\rho_{1}(z) \geq \inf \left\{|c(z)-w|: w \in S_{1}\right\}
$$

Applying Schwarz lemma to $\Theta_{z}$, we have

$$
\left.\inf \left\{\frac{\left|c(z)-\Theta_{z}(\xi)\right|}{\rho(z)}: \xi \in S_{2}\right\}=\inf \{\sigma(\vec{a}(z)), \xi): \xi \in S_{2}\right\}
$$

But $S_{1}=\Theta_{z}\left(S_{2}\right)$, and since $\rho(z) \rightarrow 1$ if $|\Pi(z)| \rightarrow I$, Theorem 1 follows from (6) and (7) if we show that

$$
\left.\inf \{\sigma(\bar{a}(z)), \xi): \xi \in S_{2}\right\} \rightarrow 1
$$

if $|\Pi(z)| \rightarrow 1$.

Suppose to the contrary that there is a sequence $\left\{v_{k}\right\} \subset D$ such that $\left|\Pi\left(v_{k}\right)\right| \rightarrow 1$ and

$$
\inf \left\{\sigma\left(\bar{\alpha}\left(v_{k}\right), \xi\right): \xi \in S_{2}\right\} \leq t<1
$$

for $k=1,2, \ldots$. Replacing $\left\{v_{k}\right\}$ by a subsequence if necessary, we may assume that $\left\{v_{k}\right\}$ is interpolating.

By Lemma 1 , the radius $\rho_{2}$ of $S_{2}$ satisfies

$$
\rho_{2}\left(v_{k}\right) \geq 1-C\left(1-\left|v_{k}\right|\right), k=1,2, \ldots
$$

By well knows properties of the metric $\sigma([2$, page 3$])$ it follows from $(8)$ and (9) that

$$
\left|a\left(v_{k}\right)\right|=\left|\frac{S\left(v_{k}\right)}{R\left(v_{k}\right)}\right| \geq 1-C_{1}\left(1-\left|v_{k}\right|\right)
$$


with $C_{1}$ depending only on $t$ and $C$. Since $\rho=\frac{|\Pi|}{|R|^{2}-|S|^{2}}$, Lemma liii gives that $1 \geq\left(|R|^{2}-|S|^{2}\right)^{-1}$. If this is combined with (10), we get

$$
\left|R\left(v_{k}\right)\right|^{2} \geq \frac{C_{1}}{1-\left|v_{k}\right|}
$$

with $C^{1}$ independent of $k$. This means that $\sum_{k}\left(1-\left|v_{k}\right|^{2}\right)\left|R\left(v_{k}\right)\right|^{2}=\infty$ contradicting that $\left\{v_{k}\right\}$ is interpolating by Lemma $1(v) v$ and the final remarks in 1 .

To prove Theorem 2, we need a lemma on Blaschke products that certainly must be well known. If $0<\alpha<\frac{\pi}{2}$, we define the cone

$$
\tau_{\alpha}=\{z:|\arg (1-z)| \leq \alpha\}
$$

Lemma 2. Let $B$ be an inner function. The following statements are equivalent

(i) $B$ is Blaschke product whose zeroes is a finite union of interpolating sequences contained in some cone $\tau_{\alpha}$.

(ii) $\inf \left\{|B(z)|, z \in D \backslash \tau_{t}\right\}>0$ for some $t$.

(iii) Given $\epsilon>0$, there is $r$ such that $|B(z)|>1-\epsilon$ if $z \in D \backslash T_{r}$.

Proof of Lemma 2: To prove (i) $\rightarrow$ (ii), we may assume that the zeroes $\left\{a_{n}\right\}$ of $B$ form an interpolating sequence. We fix $t \in\left(\alpha, \frac{\pi}{2}\right)$ and then choose $\delta>0$ so small that

$$
D_{n}=\left\{z: \sigma\left(z, a_{n}\right)<\delta\right\} \subset \tau_{\ell}, n=1,2, \ldots
$$

Let $F_{\delta}=D \bigcup_{n} D_{n}$. If follows from Carlesons condition $\left(C_{1}\right)$ in 1 that $D_{n} \cap$ $D_{m}=\phi$ if $n \neq m$ provided $\delta$ is small enough. Condition $\left(C_{1}\right)$ also give $\inf \left\{|b(z)|: z \in F_{\delta}\right\}>0$ and since $D \backslash \tau_{l} \subset F_{\delta}$, (ii) follows.

To see that (ii) $\rightarrow$ (iii), let $W$ be one of the two components of $D \backslash \tau_{t}$. Let $w(z)$ denote the harmonic measure of $\partial W \cap \partial D$ with respect to $W$, evaluated at $z \in W$. If $\epsilon>0$, it is evident that $w(z)>I-\epsilon$ in $W \backslash \tau_{r}$, if $r$ is sufficiently close to $\frac{\pi}{2}$. Since $\log |B|$ is a bounded harmonic function in $W$, (iii) follows.

To prove (iii) $\rightarrow$ (i), we first observe that any singular inner function fails to satisfy (iii). It follows that $B$ is a Blaschke product with zeroes $\left\{a_{n}\right\}$ contained in $D \cap \tau_{\alpha}$, for some $\alpha$.

Let $N_{k}$ denote the number of points from $\left\{a_{n}\right\}$ contained in $R_{k}=\tau_{a} \cap\{x$ : $\left.1-2^{-k} \leq|z|<1-2^{-k-1}\right\}$. Elementary properties of the metric $\sigma$ ([2, page 2]) shows that

$$
\inf _{z \in D \backslash \tau_{r}} \sup _{w \in R_{k}} \sigma(z, w) \leq d<I
$$

with $d$ independent of $k$. By (iii) we have

$$
|B(z)| \geq d^{N_{k}} \geq|B(z)| \geq 1-\epsilon
$$


if $z \in D \backslash \tau_{r}$. This means that $\left\{N_{k}\right\}$ is a bounded sequence. Hence we can split $\left\{a_{n}\right\}$ into finitely many sequences $\left\{b_{n}\right\}$ such that either $\left\{b_{n}\right\} \subset \tau_{\alpha} \cap\left(\bigcup_{k} R_{2 k}\right)$ or $\left\{b_{n}\right\} \subset \tau_{\alpha} \cap \cup\left(R_{2 k-1}\right)$. But then $\left\{b_{n}\right\}$ is an interpolating sequence. For details see $\left[3\right.$, ch. 10]. Alternatively, it is not hard to check that $\left(C_{1}\right)$ and $\left(C_{2}\right)$ in I holds for such a sequence $\left\{a_{n}\right\}$.

Proof of Theorem 2: We use the notation from the proof of Theorem 1 and consider a scaled problem $(N P)$ together with an expanded problem $(N P)_{1}$.

Suppose first that $(N P)_{1}$ has more than one solution. By Theorem 1 $\left|I_{\alpha}(z)\right| \rightarrow I$ if $|\Pi(z)| \rightarrow 1$ uniformly in $\alpha \in[0,2 \pi)$. Assuming $\Pi$ satisfies Lemma 2, we see that all $I_{\alpha}$ also satisfy Lemma 2 .

If $(N P)_{1}$ has a single solution $f$, we have

$$
f=\frac{P-Q I}{R-S I}
$$

where $I$ is the unique solution of the problem $(N P)_{2}$ corresponding to (5). By the classical Pick-Nevanlinna theorem $I$ is a finite Blaschke product and hence

$$
|I(z)| \geq 1-c(1-|z|), z \in D
$$

for some $c>0$. The function $f$ given above is inner $([9$, p. 492]) and by Schwartz lemma applied to $\theta_{z}$, we have again

$$
\frac{|f(z)-c(z)|}{\rho(z)}=\rho(a(\bar{z}), I(z))
$$

Hence if $f$ fail to satisfy Lemma 2, we can pick a sequence $\left\{v_{k}\right\} \subset D \backslash \tau_{r}$ such that $\rho\left(v_{k}\right) \rightarrow 1, c\left(v_{k}\right) \rightarrow 0$ and deduce

$$
\sigma\left(\bar{a}\left(v_{k}\right), I\left(v_{k}\right)\right) \leq d<1
$$

for $k=1,2, \ldots$. Combining (11) and (12) we get as before

$$
\left|\frac{S\left(v_{k}\right)}{R\left(v_{k}\right)}\right| \geq 1-C\left(1-\left|v_{k}\right|\right)
$$

and obtain a contradiction as in the proof of Theorem 1 .

Let us finally explain briefiy the proof of Theorem 3. Again we adopt the notation from the proof of Theorem 1 . Hence we consider an extremal solution

$$
f_{0}=\frac{P-Q I}{R-S I}
$$

where $P, Q, R$ and $S$ correspond to a scaled problem $(N P)$ and $I$ is a finite Blaschke product. 
Define as before $F_{\delta}=D \bigcup_{n} D_{n}$ where $D_{n}=\left\{z: \sigma\left(z, z_{n}\right)<\delta\right\}$. If $\left\{z_{n}\right\}$ is the union of $K$ interpolating sequences, it follows from Carlesons condition $\left(C_{1}\right)$ that no $z \in D$ is contained in more than $K$ different discs $D_{n}$, if $\delta$ is sufficiently small.

We fix such a small $\delta$ and assume $r \leq \frac{\delta_{1}}{10}$ in Theorem 3, where $\delta_{1}=$ $\inf \left\{|\Pi(z)|: z \in F_{\delta}\right\}>0$. If $h$ solves $(N P)$ and $\|h\|_{\infty} \leq r$, then

$$
F_{w}=h+\Pi w
$$

solves $(N P)$ whenever $|w| \leq 1-r$. If $z \in F_{\delta}$, we then have

$$
\left\{w:|w| \leq \frac{8}{10}|\Pi(z)|\right\} \subset \Delta(z) \subset\{w:|w| \leq|\Pi(z)|\} .
$$

Again we get by Schwartz lemma

$$
\left|\frac{f_{0}(z)-c(z)}{\rho(z)}\right|=\sigma(\bar{a}(z), I(z)) .
$$

If $f_{0}\left(v_{k}\right) \rightarrow 0$ for some sequence $\left\{v_{k}\right\} \subset F_{\delta}$, we get from (13) and (14) that $\sigma\left(\bar{a}\left(v_{k}\right), I\left(v_{k}\right)\right) \leq t<1, k=1,2, \ldots$ and obtain a contradiction as in the proof of Theorem 1 .

Hence the inner function $f_{0}$ must satisfy inf $\left\{\left|f_{0}(z)\right|: z \in F_{\delta},|z| \geq r\right\}>0$ for some $r \in(0,1)$. From the way $F_{b}$ was constructed, this implies that $f_{0}$ factors into finitely many interpolating Blaschke products

\section{Concluding remarks}

Consider again the problem $(N P)$ with data $\left\{z_{n}\right\},\left\{w_{n}\right\}$. Let $r_{N}$ denote the minimal norm for the truncated problem $f\left(z_{n}\right)=w_{n}, n \geq N$. Even if $r_{N} \rightarrow 0$ as $N \rightarrow \infty$, it may be the case that there is no Blaschke product among the extremal solutions to $(N P)$. In fact the singular inner function

$$
I(z)=\exp \left[-\frac{1+z}{1-z}\right]
$$

can be realized as the unique solution to such a problem.

Suppose on the other hand that there are functions $h_{N} \in H^{\infty}$ such that

$$
h_{N}\left(z_{n}\right)=\left\{\begin{array}{l}
0<n \leq N \\
w_{N}, n>N
\end{array}\right.
$$

and $\left\|h_{N}\right\|_{\infty} \rightarrow 0$. In this case we say that $(N P)$ is superscaled. Using dual extremal functions as Öyma did in [11], it is easy to see that any superscaled problem has a unique minimal solution $\lambda I$ where $\lambda$ is a constant and $I$ is inner. 
We do not know if $I$ necessarily is a Blaschke product in this case. If $\left\{z_{n}\right\}$ is a finite union of interpolating sequences, $T$. Nakazi [5] studied problems of type $(N P)$ with data $\left\{z_{n}\right\},\left\{w_{n}\right\}$, where $\frac{w_{n}}{\delta_{n}} \rightarrow 0$ and $\delta_{n}=\prod_{\substack{k=1 \\ k \neq n}}^{\infty} \sigma\left(z_{k}, z_{n}\right)$.

It is not hard to see that such problems are superscaled. In [5], Nakazi showed that the minimal solution to such a problem is a complex muitiple of an inner function. By Theorem 3 we see that this inner function is indecd a Blaschke product.

Let us finally mention an apparently difficult open problem: Is Theorem 3 valid with some numerical constant $r$ independent of $\left\{z_{n}\right\}$ ? For applications it would be sufficient to find some extremal solution satisfying the conclusion under the assumption that $\left\{z_{n}\right\}$ is an interpolating sequence.

Some recent work by A. Nicolau [8] should also be mentioned in this connection.

Let us finally give the necessary references to provide a proof of Lemma 1 .

Lemma 1 (i) and (ii) is in Nevanlinnas original paper. That (iii) holds is explained in $[9$, page 494]. If $(N P)$ is a finite problem, it follows from Nevanlinnas work that $\rho(z)$ is of class $C^{\infty}$ near the unit circle. Hence (iv) follows.

The lower bound for $\rho$ in (v), is used in [9, Lemma 2]. In [9] we also observed that $\left(R-e^{i \alpha} S\right)^{-1} \in H^{2}$ if $0 \leq \alpha<2 \pi$ and $(N P)$ has more than one solution. If $(N P)$ is scaled, one even has $\left(R-e^{i \alpha} S\right) \in H^{2}$. This is seen from Theorem 4.4 and Theorem 5.5 in Ch. IV in Garnetts book [2], and observing, that the function $F$ in Lemma 5.6 corresponds to $\left(R-e^{i \alpha} S\right)^{-2}$. But then (v) must hold.

\section{References}

1. L CARLeson, An interpolation problem for bounded analytic functions, Amer. J. Math. 80 (1958), 921-930.

2. J. Garnetr, "Bounded analytic funciions," Academic Press, London, 1981.

3. K. Hofrman, "Banach spaces of analytic functions," Prentice Hall, Englewood Cuths N.J., 1962.

4. P. JoNEs, Estimates for the corona problem, J. Funct. Anal. 39 (1980), 162-181.

5. T. NAKAZI, Notes on interpolation by bounded analytic functions, Canad. Math. Bull. 31 (4) (1988).

6. R. Nevanlinna, Über bechränkte analytiscke Functionen, Ann. Acad. Sci Fenn. 32, 7 (1929).

7. A. NiCOLAU, Sobre el problema d'interpolació de Pick-Nevanlinna, Tesi, Universitad Autònoma de Barcelona, 1989. 
8. A. NicOlaU, Solving Pick-Nevanlinna problems by interpolating Blaschke products, (Preprint).

9. A. STray, Minimal interpolation by Blaschke products, J. London Math. Soc. (2) 32 (1985), 488-496.

10. A. STray, Minimal interpolation by Blaschke products II, Bull. London Math. Soc. 20 (1988), 329-332.

11. K. ÖYMA, Extremal interpolating functions in $H^{\infty}$, Proc. Amer. Math. Soc. 64 (1977), 272-276.

\author{
Universitetet i Bergen \\ Matematisk Institutt \\ Allégt. 55 \\ N-5007 Bergen \\ NORGE
}

Primera versió rebuda el 23 d'Agost de 1990 , dartera versió rebuda el 15 de Gener de 1991 